\title{
Human Interface for Maneuvering Nonholonomic Systems
}

\author{
Hirohiko Arai \\ Intelligent Systems Institute \\ National Institute of Advanced Industrial Science and Technology \\ 1-2 Namiki, Tsukuba, Ibaraki 305-8564, Japan \\ Email: h.arai@aist.go.jp
}

\begin{abstract}
Humans can easily maneuver some types of nonholonomic systems, e.g. wheeled vehicles, while other types, e.g. space robots, are difficult to handle intuitively. We propose a human interface to simplify the operation of "difficult" nonholonomic systems, which utilizes the human ability to maneuver "easy" systems. The difficult real system is converted into an easy virtual system using coordinate and input transformation. The input from the human operator to the virtual system is converted into input to the real system, while the state of the real system is converted into that of the virtual system which is displayed to the operator. The operator can then steer the real system feeling as if maneuvering the virtual system. Our experiments show that the operating performance is improved by this method.
\end{abstract}

\section{Introduction}

Control of nonholonomic systems has become a popular topic in robot control over the past ten years [1]. A mechanical constraint which cannot be represented as an algebraic equation, $\boldsymbol{g}(\boldsymbol{q}, t)=\mathbf{0}(t$ : time, $\boldsymbol{q}$ : generalized coordinate), is called a nonholonomic constraint. Robotics researchers mainly deal with nonholonomic systems with nonintegrable velocity constraints. Typical examples of such constraints are kinematic constraints with rolling contact, e.g. wheeled vehicles $[2,3]$ and nonholonomic manipulators [4], and dynamic constraints due to conservation of angular momentum, e.g. space robots [5, 6].

In these examples, the constraint is represented as a Pfaffian form,

$$
\boldsymbol{h}(q) \dot{q}=\mathbf{0}
$$

and the state equation is in a drift-free affine form,

$$
\dot{q}=G(q) u
$$

These types of nonholonomic systems have the following characteristics;

(a) The system is often controllable and the system can reach any configuration.

(b) The components of the input $\boldsymbol{u}$ are fewer than those of the state $\boldsymbol{q}$.

(c) The linear approximation of the system (2) is uncontrollable.

(d) There exists no time-invariant state feedback law that stabilizes the system to an equilibrium state (Brockett's theorem [7]).

Although (a) suggests the possibility of control, it involves difficulties such as (b), (c) and (d). On the contrary, such difficulties of nonholonomic systems attract researchers' interest in challenging theoretical problems rather than practical requirements. Therefore, most of the control methods proposed so far have aimed at complete automation where no human intervenes.

As described above, control of nonholonomic systems is generally a difficult problem for a robot or a computer. However, there is controversy over whether all nonholonomic systems are difficult to control for a human. A wheel is one of the oldest inventions of humankind and has a long history from ancient times. We know that most people can commonly steer nonholonomic vehicles such as a bicycle, automobile and pushcart, even though some practice is necessary. On the other hand, we may assume that a space robot would be very difficult to operate for a human because we have no chance encounter such systems in our daily life. Thus, the nonholonomic systems of Eq. (2) include two types, i.e. "easy" systems and "difficult" systems for a human to maneuver.

There have been few studies in robotics about manmachine systems with nonholonomic constraints. Colgate et al. [8] developed a haptic display using a nonholonomic mechanism. They also proposed applying 
the same mechanism to a motion guide in humanrobot collaboration [9, 10]. Tanaka et al. [11] analyzed the behavior of a human upper-limb when a nonholonomic constraint is applied. We proposed a virtual nonholonomic constraint for human-robot cooperative manipulation of a long object [12]. However, these studies do not directly intend to help a human to maneuver nonholonomic systems.

In this paper, we propose a human interface to aid in maneuvering nonholonomic systems. We utilize the human ability of handling "easy" nonholonomic systems for the human operation of "difficult" systems. This method would be useful in the realtime teleoperation and manual off-line programming of nonholonomic systems such as space robots. Moreover, the human's adaptability in the control loop enables us to construct a robust system.

The rest of this paper is organized as follows. In Section 2, we describe experiments on human operation of two typical nonholonomic systems. We show that there exist easy systems and difficult systems for a human to maneuver. In Section 3, we propose an interface that helps a human to maneuver the difficult systems. The "difficult" real system is converted into an "easy" virtual system using coordinate and input transformation. The human operator apparently controls the virtual system while operating the real system. In Section 4, we experimentally demonstrate the effectiveness on proposed method.

\section{Human ability to maneuver nonholo- nomic systems}

In this section, we describe experiments we conducted in which the human operators maneuver two typical nonholonomic systems, a single wheel and a space robot, in a simulator. By comparing those maneuvering characteristics, we verified that the difficulty in human operation varies depending on the system.

First, we deal with a single wheel as a nonholonomic system which can be expected to be easy to maneuver for a human (Fig. 1). The state equation is represented as;

$$
\left(\begin{array}{c}
\dot{x} \\
\dot{y} \\
\dot{\theta}
\end{array}\right)=\left(\begin{array}{cc}
\cos \theta & 0 \\
\sin \theta & 0 \\
0 & 1
\end{array}\right)\left(\begin{array}{l}
v \\
\omega
\end{array}\right)
$$

The control inputs to the system (3) are forward/ backward velocity $v$ and angular velocity $\omega$. Since we use a computer mouse for the input device, we modify

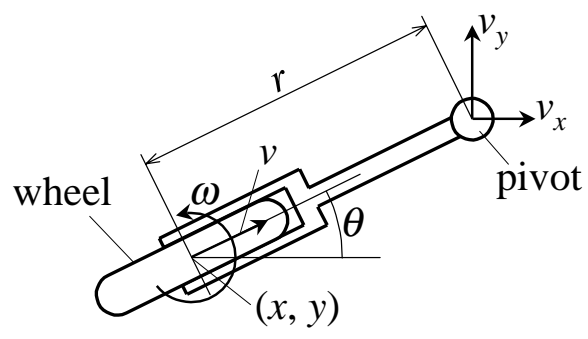

Fig. 1: Model of single wheel

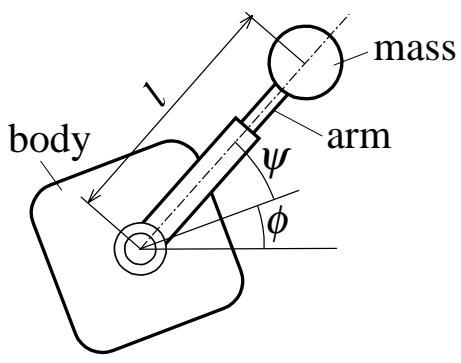

Fig. 2: Model of space robot

these inputs to allow an intuitive maneuver. We attached an arm of length $r$ in the direction of the wheel. The translational velocity input $\left(v_{x}, v_{y}\right)$ is given to the pivot at the end of the arm. The operator can then steer the wheel just like a wheelbarrow. The velocity inputs to Eq. (3) are;

$$
\left\{\begin{aligned}
v & =v_{x} \cos \theta+v_{y} \sin \theta \\
\omega & =\left(-v_{x} \sin \theta+v_{y} \cos \theta\right) / r
\end{aligned}\right.
$$

$\left(v_{x}, v_{y}\right)$ is given proportional to the velocity of the mouse.

On the other hand, we consider a free-flying space robot as an example of a difficult system for a human to operate. The robot is a simple planar model in Fig. 2. The arm has a rotational and a prismatic joints. The rotational axis of the arm coincides with the center of the body mass. The arm is assumed to be massless and has a point mass of $M$ at its end. The moment of inertia of the body about the center of mass is $I$, and the length of the arm is $l$. The orientation of the body in the absolute frame is $\phi$, and the angle of the arm relative to the body is $\psi$. The initial angular momentum is assumed to be zero. The state 
equation of this space robot is;

$$
\left(\begin{array}{l}
\dot{\psi} \\
\dot{l} \\
\dot{\phi}
\end{array}\right)=\left(\begin{array}{cc}
1 & 0 \\
0 & 1 \\
-\frac{M l^{2}}{I+M l^{2}} & 0
\end{array}\right)\left(\begin{array}{l}
\eta \\
\lambda
\end{array}\right)
$$

The control inputs are the angular velocity of the arm relative to the body, $\eta$, and the stretching velocity of the arm, $\lambda$.

The movements of the arm end and the computer mouse are synchronized. When the translation of 1 body due to the arm motion is neglected, the posit: of the arm end is;

$$
\left\{\begin{array}{l}
x=l \cos (\phi+\psi) \\
y=l \sin (\phi+\psi)
\end{array}\right.
$$

in the absolute frame. The velocity of the arm end

$$
\left\{\begin{array}{l}
v_{x}=i \cos (\phi+\psi)-l(\dot{\phi}+\dot{\psi}) \sin (\phi+\psi) \\
v_{y}=i \sin (\phi+\psi)+l(\dot{\phi}+\dot{\psi}) \cos (\phi+\psi)
\end{array}\right.
$$

From Eq. (5),

$$
\dot{\phi}+\dot{\psi}=\frac{I}{I+M l^{2}} \eta, \quad i=\lambda
$$

Then,

$$
\left\{\begin{array}{l}
\eta=-\frac{I+M l^{2}}{I l}\left\{v_{x} \sin (\phi+\psi)-v_{y} \cos (\phi+\psi)\right\} \\
\lambda=v_{x} \cos (\phi+\psi)+v_{y} \sin (\phi+\psi)
\end{array}\right.
$$

$\left(v_{x}, v_{y}\right)$ is proportional to the velocity of the mous

The human operators maneuvered the single wh and the space robot in a simulator. The input dev was a computer mouse, and the display device a $\mathrm{C}$ display. The sampling period for the input and disp. is 20 msec. The current and desired states of the ject were displayed on the CRT and the operators w instructed to position the object to the desired sta The operators were four males in their $20 \mathrm{~s}$. One tr continued for 20 sec. After practicing several tim the data of five trials were recorded.

The displayed image of the single wheel is sho in Fig. 3. The operators steer the position and ori, tation of the wheel (large circle) by moving the pi (small circle) using the mouse. The initial state the wheel is $\left(x_{0}, y_{0}, \theta_{0}\right)=(0.181,0.930, \pi / 4)$ and 1 desired state is $\left(x_{d}, y_{d}, \theta_{d}\right)=(0.529,0,0)$.

Fig. 4 shows the displayed image of the space rob The operators move the end of the arm to maneu the configuration of the arm and the body. The iner parameters of the robot are $M=1$ and $I=2$. T workspace of the arm is limited within $1<l<2$ a $-\pi / 2<\psi<\pi / 2$. These limits are also display

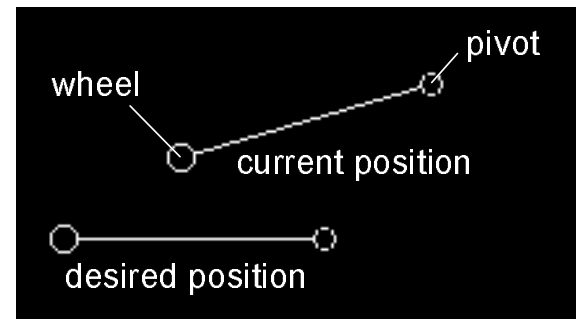

Fig. 3: Displayed image of wheel

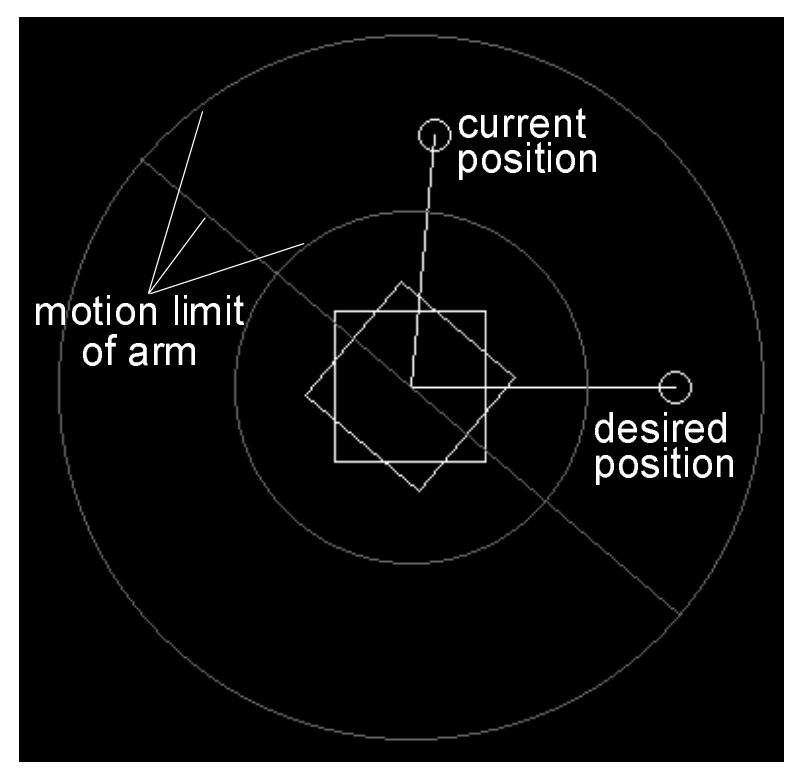

Fig. 4: Displayed image of space robot

The initial state of the space robot is $\left(\phi_{0}, \psi_{0}, l_{0}\right)=$ $(\pi / 4, \pi / 4,1.5)$ and the desired state is $\left(\phi_{d}, \psi_{d}, l_{d}\right)=$ $(0,0,1.5)$.

The experimental data were evaluated with the minimum square error to the desired state, min(error ${ }^{2}$, the mean square error during the experiment, $\frac{1}{T} \int_{0}^{T}$ error $^{2} d t$, and the time interval, $T_{e}$, until the square error was reduced below the threshold value. The square error is calculated as $e_{x y \theta}^{2}=$ $\left(x-x_{d}\right)^{2}+\left(y-y_{d}\right)^{2}+\left(\theta-\theta_{d}\right)^{2}$ for the single wheel, and as $e_{\phi \psi l}^{2}=\left(\phi-\phi_{d}\right)^{2}+\left(\psi-\psi_{d}\right)^{2}+\left(l-l_{d}\right)^{2}$ for the space robot.

An example of the positioning of the single wheel is shown in Fig. 5. Table 1 shows the evaluated data for each operator. The data are the average of five trials. The threshold for the positioning is $e_{x y \theta}^{2}<0.001$. All of the operators finished positioning the wheel within 6 sec and it reached the desired configuration precisely. The operators hardly required practice before under- 


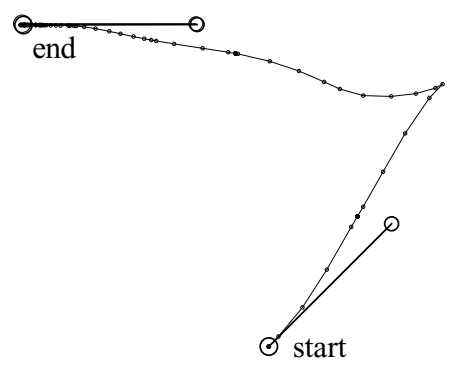

Fig. 5: Positioning of wheel

Table 1: Experimental results (wheel)

\begin{tabular}{|c|c|c|c|}
\hline Operator & $\min \left(e_{x y \theta}^{2}\right)$ & $\frac{1}{T} \int_{0}^{T} e_{x y \theta}^{2} d t$ & $T_{e}(\mathrm{sec})$ \\
\hline $\mathrm{A}$ & $1.19 \times 10^{-5}$ & 0.207 & 5.64 \\
\hline $\mathrm{B}$ & $2.21 \times 10^{-5}$ & 0.250 & 5.88 \\
\hline $\mathrm{C}$ & $2.54 \times 10^{-5}$ & 0.206 & 4.24 \\
\hline $\mathrm{D}$ & $3.03 \times 10^{-5}$ & 0.155 & 4.12 \\
\hline
\end{tabular}

standing how to steer the wheel to the desired state. It was not necessary to explain the behavior of the wheel to the operators.

In contrast, none of the operators could rotate the body of the space robot at first. The basic behaviors of the robot were additionally explained to the operators: the arm rotation causes the reverse rotation of the body due to the reaction torque, and that the body rotation increases according to the arm length. After the explanation, the operators could understand that the circular motion of the arm end results in the body rotation, and they could operate the robot close to the desired state. However, the operators sometimes rotated the body in reverse direction, missing the direction of the circular motion of the arm. The fine rotation of the body near the desired state was very difficult, because the operators could not know the relation between the size of the arm motion and the angle of the body rotation. Fig. 6 shows an example of the positioning of the space robot. Table 2 shows the averaged evaluation data for each operator. The threshold for the positioning is $e_{\phi \psi l}^{2}<0.001$.

The numerical data cannot be compared simply because the structures of the systems are quite different. However, it is evident that the space robot was much more difficult to maneuver than the single wheel. There are several reasons for this difficulty. First, a human does not naturally have the skill to operate a space robot because such a system is not encountered in ordinary life. Next, the arm motion and the

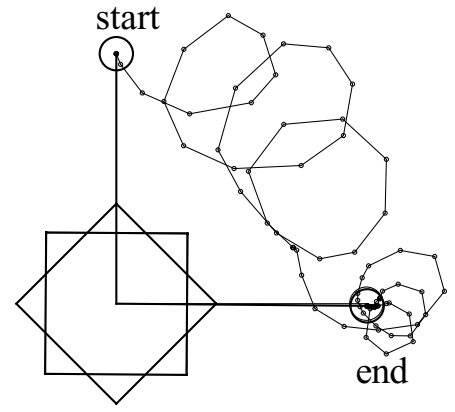

Fig. 6: Positioning of space robot

Table 2: Experimental results (space robot)

\begin{tabular}{|c|c|c|c|}
\hline Operator & $\min \left(e_{\phi \psi l}^{2}\right)$ & $\frac{1}{T} \int_{0}^{T} e_{\phi \psi l}^{2} d t$ & $T_{e}(\mathrm{sec})$ \\
\hline $\mathrm{A}$ & $1.92 \times 10^{-4}$ & 0.219 & 8.58 \\
\hline $\mathrm{B}$ & $1.04 \times 10^{-4}$ & 0.552 & 13.04 \\
\hline $\mathrm{C}$ & $4.49 \times 10^{-4}$ & 0.720 & 15.14 \\
\hline $\mathrm{D}$ & $2.26 \times 10^{-4}$ & 0.240 & 12.06 \\
\hline
\end{tabular}

body rotation are related through the inertial parameters, $M$ and $I$. Those parameters were not visually expressed and the operators could not make strategies to maneuver the robot based on a displayed image. In case of the single wheel, the operators could visually understand the direction of the constraint, and they could plan the operation predicting the response to the input.

\section{Human interface via system trans- formation}

In the previous section, we verified that there are both easy and difficult nonholonomic systems for human to maneuver, even though they are both represented as Eq. (2). Here we propose a human interface to maneuver the difficult systems utilizing the human skill to deal with the easy systems.

\subsection{Transformation into virtual system}

Let us consider two nonholonomic systems,

$$
\begin{array}{ll}
\dot{\boldsymbol{q}}=\boldsymbol{G}(\boldsymbol{q}) \boldsymbol{u} & \left(\boldsymbol{q} \in \Re^{n}, \boldsymbol{u} \in \Re^{m}, n>m\right) \\
\dot{\boldsymbol{x}}=\boldsymbol{H}(\boldsymbol{x}) \boldsymbol{v} & \left(\boldsymbol{x} \in \Re^{n}, \boldsymbol{v} \in \Re^{m}, n>m\right)
\end{array}
$$

The numbers of the states, $\boldsymbol{q}$ and $\boldsymbol{x}$, and the numbers of the inputs, $\boldsymbol{u}$ and $\boldsymbol{v}$, are same, respectively. 
We assume that system (10) and system (11) can be converted to each other with the coordinate transformation,

$$
x=\alpha(q)
$$

and the input transformation,

$$
\boldsymbol{v}=\boldsymbol{\beta}(q) \boldsymbol{u}
$$

Where, $\frac{\partial \boldsymbol{\alpha}}{\partial \boldsymbol{q}}$ and $\boldsymbol{\beta}$ are nonsingular. Substituting Eqs. (12) and (13) into Eq. (11),

$$
\frac{\partial \boldsymbol{\alpha}}{\partial \boldsymbol{q}} \dot{\boldsymbol{q}}=\boldsymbol{H}(\boldsymbol{\alpha}(q)) \boldsymbol{\beta}(q) \boldsymbol{u}
$$

From Eq. (10) and the above equation,

$$
\frac{\partial \boldsymbol{\alpha}}{\partial q} \boldsymbol{G}(q)=\boldsymbol{H}(\boldsymbol{\alpha}(q)) \boldsymbol{\beta}(q)
$$

If there exist $\boldsymbol{\alpha}(\boldsymbol{q})$ and $\boldsymbol{\beta}(q)$ that satisfy this equation, systems (10) and (11) are equivalent and can be mutually converted.

Here, system (10) is assumed to be easy to maneuver for a human, while system (11) is difficult. We consider the problem of a human steering the system (11) to the desired state $\boldsymbol{x}_{d}$. The current state $\boldsymbol{x}$ and the desired state $\boldsymbol{x}_{d}$ of the real system (11) are converted into the current state $\boldsymbol{q}$ and the desired state $\boldsymbol{q}_{d}$ of the virtual system (10), respectively, using the inverse transformation of Eq. (12),

$$
q=\alpha^{-1}(x)
$$

and are displayed to the operator. In addition, the inequality constraints such as obstacles and motion limits on system (11) are similarly converted into the constraints on system (10) and displayed. On the other hand, the input $\boldsymbol{u}$, which the operator gives to system (10) based upon the displayed state, is converted into the input $\boldsymbol{v}$ to system (11) according to Eq. (13) (Fig. 7). The operator can then maneuver the real system (11) feeling as if he or she were operating the virtual system (10). When the virtual system (10) reaches the desired state $\boldsymbol{q}_{d}$, the real system (11) also reaches the desired state $\boldsymbol{x}_{d}$.

This method is similar to the operational space controller of a robot manipulator. It is difficult for a human to position the tip of the manipulator by controlling each joint. However, a human can intuitively maneuver the manipulator by converting the cartesian position command into the joint angles while watching the tip position in the cartesian frame.

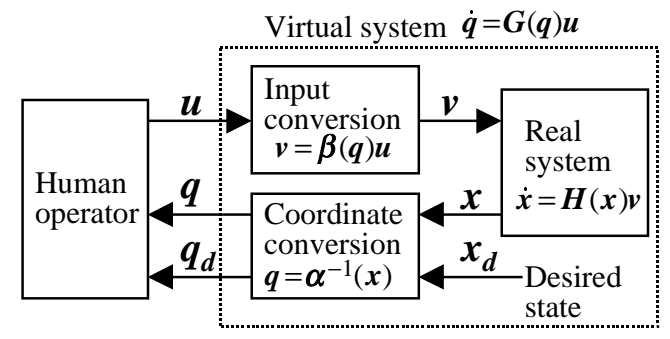

Fig. 7: Human interface via system transformation

\subsection{Transformation via canonical form}

It is a difficult problem to find $\boldsymbol{\alpha}(\boldsymbol{q})$ and $\boldsymbol{\beta}(q)$ from Eq. (15) for the conversion between general nonholonomic systems. Hence, we considered system transformation through a chained form, which is often studied as a canonical form for nonholonomic systems [2]. Let us assume that two nonholonomic systems with two inputs,

$$
\begin{array}{ll}
\dot{\boldsymbol{q}}=\boldsymbol{g}_{1}(\boldsymbol{q}) u_{1}+\boldsymbol{g}_{2}(\boldsymbol{q}) u_{2} & \left(\boldsymbol{q} \in \Re^{n}, \quad u_{1}, u_{2} \in \Re\right) \\
\dot{\boldsymbol{x}}=\boldsymbol{h}_{1}(\boldsymbol{x}) v_{1}+\boldsymbol{h}_{2}(\boldsymbol{x}) v_{2} & \left(\boldsymbol{x} \in \Re^{n}, \quad v_{1}, v_{2} \in \Re\right)
\end{array}
$$

can be converted into an identical chained system,

$$
\left\{\begin{array}{ccc}
\dot{\xi}_{1} & = & \nu_{1} \\
\dot{\xi}_{2} & =\nu_{2} \\
\dot{\xi}_{3} & =\xi_{2} \nu_{1} \\
& \vdots \\
\dot{\xi}_{n} & =\xi_{n-1} \nu_{1}
\end{array}\right.
$$

using the coordinate and input transformations,

$$
\begin{aligned}
& \boldsymbol{\xi}=\boldsymbol{\alpha}_{1}(\boldsymbol{q}), \quad\left(\begin{array}{l}
\nu_{1} \\
\nu_{2}
\end{array}\right)=\boldsymbol{\beta}_{1}(\boldsymbol{q})\left(\begin{array}{l}
u_{1} \\
u_{2}
\end{array}\right) \\
& \boldsymbol{\xi}=\boldsymbol{\alpha}_{2}(\boldsymbol{x}), \quad\left(\begin{array}{l}
\nu_{1} \\
\nu_{2}
\end{array}\right)=\boldsymbol{\beta}_{2}(\boldsymbol{x})\left(\begin{array}{l}
v_{1} \\
v_{2}
\end{array}\right)
\end{aligned}
$$

where, $\boldsymbol{\xi}=\left(\xi_{1}, \ldots, \xi_{n}\right)^{T}$.

From Eqs. (20) and (21),

$$
\begin{gathered}
\boldsymbol{\alpha}_{1}(\boldsymbol{q})=\boldsymbol{\alpha}_{2}(\boldsymbol{x}) \\
\boldsymbol{\beta}_{1}(\boldsymbol{q})\left(\begin{array}{c}
u_{1} \\
u_{2}
\end{array}\right)=\boldsymbol{\beta}_{2}(\boldsymbol{x})\left(\begin{array}{l}
v_{1} \\
v_{2}
\end{array}\right)
\end{gathered}
$$

Then, the coordinate and input conversions corresponding to Eqs. (12) and (13) can be obtained as;

$$
x=\alpha_{2}^{-1}\left(\alpha_{1}(q)\right)
$$




$$
\left(\begin{array}{l}
v_{1} \\
v_{2}
\end{array}\right)=\boldsymbol{\beta}_{2}(\boldsymbol{x})^{-1} \boldsymbol{\beta}_{1}(q)\left(\begin{array}{l}
u_{1} \\
u_{2}
\end{array}\right)
$$

Murray and Sastry [2] showed sufficient conditions when there exist the coordinate and input transformations (20) that convert the nonholonomic system (17) into the chained form (19). It is well known that many types of nonholonomic systems, e.g. a single wheel, differential two-wheel robot, car-like four-wheel robot [2], trailer [3], nonholonomic manipulator [4] and space robot $[5,6]$, can be equivalent to a chained form. The method in this section can be used to transform among these nonholonomic systems.

\section{Experiments}

We applied the method described in Section 3 to the two examples in Section 2 and constructed an interface for human operation. The control object is the space robot, which was difficult to maneuver in the experiments in Section 2. We transformed it into a virtual single wheel, which is easy to handle, through a chained form. The virtual wheel is used for the human interface of display and input. We conducted experiments operating the space robot and demonstrated that the operation performance was improved compared with the direct operation in Section 2.

\subsection{Conversion between space robot and single wheel}

First, we convert the single wheel system of Eq. (3) into a chained form by coordinate transformation,

$$
\left\{\begin{array}{l}
\xi_{1}=\theta \\
\xi_{2}=x \cos \theta+y \sin \theta \\
\xi_{3}=x \sin \theta-y \sin \theta
\end{array}\right.
$$

and input transformation,

$$
\left\{\begin{array}{l}
\nu_{1}=\omega \\
\nu_{2}=v-\xi_{3} \omega
\end{array}\right.
$$

Differentiating Eq. (26) and substituting Eqs. (3) and (27), a 3 -state 2 -input chained form,

$$
\left\{\begin{array}{l}
\dot{\xi}_{1}=\nu_{1} \\
\dot{\xi}_{2}=\nu_{2} \\
\dot{\xi}_{3}=\xi_{2} \nu_{1}
\end{array}\right.
$$

is obtained.

On the other hand, the space robot system of Eq. (5) can be converted into the same chained form (28) by coordinate transformation,

$$
\left\{\begin{array}{l}
\xi_{1}=\psi \\
\xi_{2}=-\frac{M l^{2}}{I+M l^{2}} \\
\xi_{3}=\phi
\end{array}\right.
$$

and input transformation,

$$
\left\{\begin{array}{l}
\nu_{1}=\eta \\
\nu_{2}=-\frac{2 M I l}{\left(I+M l^{2}\right)^{2}} \lambda
\end{array}\right.
$$

From Eq. (26),

$$
\left\{\begin{array}{l}
x=\xi_{2} \cos \xi_{1}+\xi_{3} \sin \xi_{1} \\
y=\xi_{2} \sin \xi_{1}-\xi_{3} \cos \xi_{1} \\
\theta=\xi_{1}
\end{array}\right.
$$

The current state $(\phi, \psi, l)$ and the desired state $\left(\phi_{d}, \psi_{d}, l_{d}\right)$ of the space robot are converted into the current state $(x, y, \theta)$ and the desired state $\left(x_{d}, y_{d}, \theta_{d}\right)$ of the single wheel by Eqs. (29) and (31), and are displayed to the operator. The motion limit of the arm, $\psi_{\min }<\psi<\psi_{\max }, l_{\min }<l<l_{\max }$ is also converted as,

$$
\left\{\begin{array}{c}
\psi_{\min }<\theta<\psi_{\max } \\
-\frac{M l_{\max }^{2}}{I+M l_{\max }^{2}}<x \cos \theta+y \sin \theta<-\frac{M l_{\min }^{2}}{I+M l_{\min }^{2}}
\end{array}\right.
$$

and displayed.

From Eq. (30),

$$
\left\{\begin{array}{l}
\eta=\nu_{1} \\
\lambda=-\frac{\left(I+M l^{2}\right)^{2}}{2 M I} \nu_{2}
\end{array}\right.
$$

The input $(v, \omega)$ to the single wheel is converted into the input $(\eta, \lambda)$ to the space robot according to Eqs. $(27)$ and $(33)$.

The above system transformation enables the operator to maneuver the space robot feeling as though he or she were steering the single wheel. This method can be applied to any nonholonomic system that can be converted into a 3 -state 2 -input chained form (28).

\subsection{Experimental results}

Fig. 8 shows the image displayed to the operator. In addition to the wheel and the arm, the motion limit of the wheel corresponding to that of the space robot was displayed. The velocity input proportional to the mouse velocity was given to the pivot. The data of five trials for $20 \mathrm{sec}$ were recorded for each of the four operators in the same way as in Section 2. The initial and desired states are $\left(\phi_{0}, \psi_{0}, l_{0}\right)=(\pi / 4, \pi / 4,1.5)$ and $\left(\phi_{d}, \psi_{d}, l_{d}\right)=(0,0,1.5)$, respectively. Fig. 9 shows 


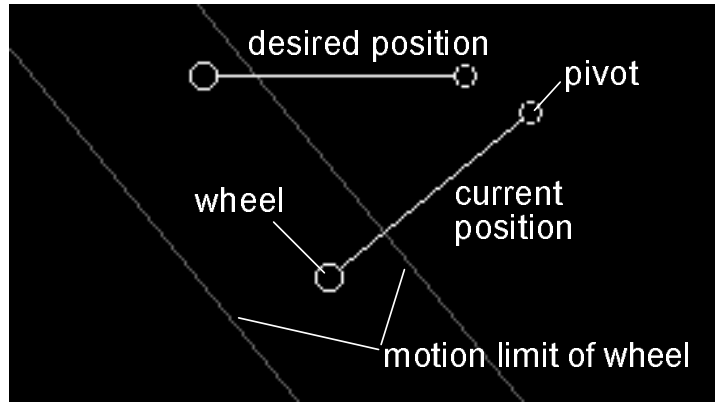

Fig. 8: Displayed image of proposed interface

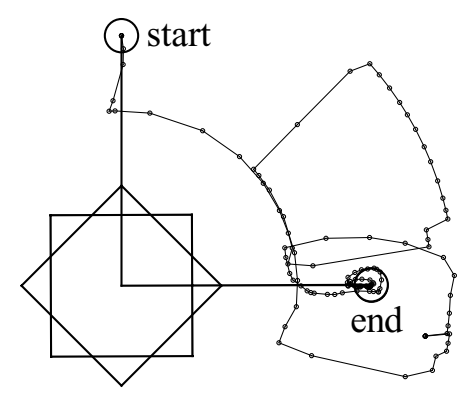

Fig. 9: Positioning with proposed interface

an example of the positioning operation. We evaluated the minimum square error, $\min \left(e_{\phi \psi l}^{2}\right)$, the mean square error, $\frac{1}{T} \int_{0}^{T} e_{\phi \psi l}^{2} d t$ and the reaching interval, $T_{e}$, before $e_{\phi \psi l}^{2}<0.001$. Table 3 shows the data for each operator. Fig. 10 shows a comparison of the results of this method and the direct operation in Section 2.

The proposed interface leads to apparently the same operation as the single wheel. The operators were able to understand immediately how to move the object to the desired state. Fine positioning near the desired state was also easy. The comparison in Fig. 10 shows improvements in the precision and quickness of the positioning, even though there were some individual variations.

Table 3: Experimental results (proposed interface)

\begin{tabular}{|c|c|c|c|}
\hline Operator & $\min \left(e_{\phi \psi l}^{2}\right)$ & $\frac{1}{T} \int_{0}^{T} e_{\phi \psi l}^{2} d t$ & $T_{e}(\mathrm{sec})$ \\
\hline $\mathrm{A}$ & $6.98 \times 10^{-5}$ & 0.395 & 10.44 \\
\hline $\mathrm{B}$ & $5.17 \times 10^{-5}$ & 0.464 & 11.00 \\
\hline $\mathrm{C}$ & $1.15 \times 10^{-4}$ & 0.309 & 9.50 \\
\hline $\mathrm{D}$ & $7.42 \times 10^{-5}$ & 0.423 & 6.54 \\
\hline
\end{tabular}

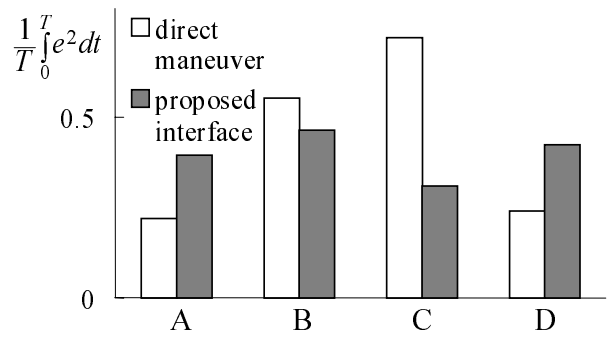

(a) mean square error

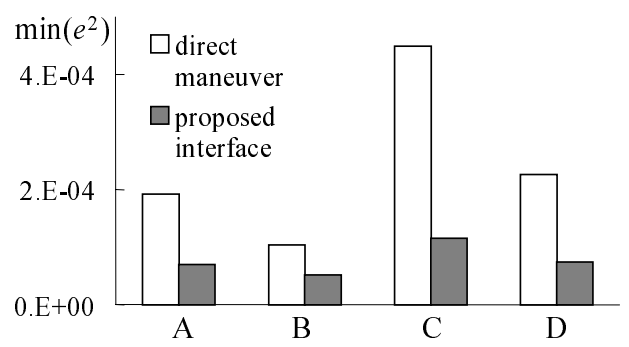

(b) minimum square error

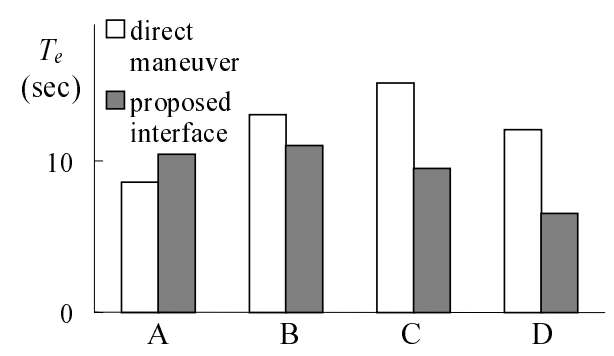

(c) positioning interval

Fig. 10: Proposed interface vs. direct maneuver

\section{Conclusions}

We proposed a method to convert a nonholonomic system difficult to operate for a human, e.g. a space robot, into an easy system to maneuver, e.g. a wheel, and use it as a human interface for display and input. The future subjects for this study are as follows;

Here, we empirically chose a single wheel as an easy nonholonomic system. We have to consider the difficulty criteria for operating more general nonholonomic systems including systems with more states and inputs, so that we can find what types of nonholonomic systems are easy to maneuver.

We converted the real nonholonomic system into a virtual system through a chained form. A transformation without using the chained form is necessary to apply this method to a wider class of nonholonomic systems. 


\section{References}

[1] I. Kolmanovsky and N. H. McClamroch: "Developments in Nonholonomic Control Problems," IEEE Control Systems, vol. 15, no. 6, pp. 20-36, 1995.

[2] R. M. Murray and S. S. Sastry: "Nonholonomic Motion Planning: Steering Using Sinusoids," IEEE Trans. Automatic Control, vol.38, no.5, pp.700-716, 1993.

[3] O. J. Sørdalen, "Conversion of the Kinematics of a Car with n Trailers into a Chained Form," Proc. of 1993 IEEE Int. Conf. on Robotics and Automation, Vol. 1, pp.382-387, 1993.

[4] O. J. Sørdalen, Y. Nakamura and W. J. Chung: "Design of a nonholonomic manipulator", Proc. of 1994 IEEE Int. Conf. on Robotics and Automation, Vol. 1, pp. 8-13, 1994.

[5] M. Sampei, H. Kiyota and M. Ishikawa: "Timestate Control Form and Its Application to a Nonholonomic Space Robot," Proc. of IFAC Symposium on Nonlinear Control Systems Design 1995, pp.679-684, 1995 .

[6] F. Matsuno and J. Tsurusaki: "Chained form transformation algorithm for a class of 3 -states and 2-inputs nonholonomic systems and attitude control of a space robot," Proc. of 38 th IEEE Conf. on Decision and Control, pp.2126-2131, 1999.

[7] R. W. Brockett: "Asymptotic Stability and Feedback Stabilization," Differential Geometric Control Theory (R. W. Brockett, R. S. Millman, and H. J. Sussmann, eds.), pp. 181-191, Birkhäuser, Boston, 1983.

[8] J. E. Colgate, M. Peshkin and W. Wannasuphoprasit: "Nonholonomic Haptic Display," Proc. of 1996 IEEE Int. Conf. on Robotics and Automation, pp. 539-544, 1996.

[9] W. Wannasuphoprasit, R. Gillespie, J. E. Colgate and M. Peshkin: "Cobot control," Proc. of 1997 IEEE Int. Conf. on Robotics and Automation, pp. $3571-3576,1997$.

[10] K. M. Lynch and C. Liu: "Designing Motion Guides for Ergonomic Collaborative Manipulation," Proc. of 2000 IEEE Int. Conf. on Robotics and Automation, pp. 2709-2715, 2000.
[11] Y. Tanaka, T. Tsuji and M. Kaneko: "Trajectory Formation of Human Arm with Nonholonomic Constraints," Proc. of 3rd Int. Conf. on Advanced Mechatronics (ICAM'98), Vol. 2, pp. $1-6,1998$.

[12] H. Arai, T. Takubo, Y. Hayashibara and K. Tanie: "Human-robot cooperative manipulation using a virtual nonholonomic constraint", Proc of 2000 IEEE Int. Conf. on Robotics and Automation, pp. 4063-4069, 2000. 